\section{Dual-Beam Microstrip Leaky-Wave Array Excited by Aperture-Coupling Method}

\author{
Tai-Lee Chen and Yu-De Lin
}

\begin{abstract}
This paper describes the design of the microstrip leaky-wave array excited by the aperture-coupling technique. The microstrip first higher order leaky mode is employed as the radiation source. Separating the radiators from other components with the ground planes provides optimal design of radiators and others so that the inherent broadband, high gain and frequency-scanning properties of the leaky-wave antenna can be exploited. Four kinds of feeding arrangements to excite dual beams are experimented in $\mathrm{K}$ band. Markedly reducing the required elements than patch-array for high gain design can simplify complexity of the feeding layout. Versatile designs combining the merits of multilayer technology offer simplicity and efficient design for practical wireless applications.
\end{abstract}

Index Terms-Aperture coupling, leaky wave, microstrip antenna.

\section{INTRODUCTION}

Broadband services such as broadband wireless access (BWA), local multipoint distribution service (LMDS), and multipoint multichannel distribution service (MMDS) are popular in wireless communications. The spectrum used in these applications is allocated in the millimeter wave frequency range. In addition, surveillance applications such as radar sensor, automobile collision avoidance system and toll and traffic management system are also operated in the microwave and millimeter wave frequency range. In these wireless applications, directional antenna and multibeam antenna play important roles [1].

Printed-circuit type antenna is the trend in antenna development, which is compact and relatively inexpensive. In millimeter wave and wide band applications, resonant type antennas, such as patch and dipoles, are usually not suitable due to their narrow bandwidth, complexity in the matching network design for array applications, and serious tolerance requirement in fabrication. Printed circuit type leaky wave antenna [2]-[5] is a better candidate in millimeter wave applications owing to its merits such as simplicity in array design, broadband, and beam-scanning capability.

The dual-beam pattern can be easily implemented by leaky-wave antenna without complicated feeding networks. The feeding structures to excite the microstrip leaky mode usually require extra matching or transition circuit [2], [3]. These circuits, however, would generate undesired fields that will affect the current distribution of the antenna mode and contaminate the radiation pattern. These extra circuits might also limit the bandwidth. First proposed by Pozar as the feeding for the patch antenna [6], aperture coupling can avert these drawbacks by separating the radiators from other networks with a conductor plane. This technique can be used to design optimally for both antenna and other circuits [7].

\section{Design OF THE Microstrip FIRST Higher ORder LEAKY-WAVE ARRAY EXCITED BY THE APERTURE-COUPLING METHOD}

Fig. 1 illustrates four kinds of feeding arrangements for dual-beam aperture-coupled microstrip leaky-wave array. The feeding microstrip

Manuscript received February 19, 1999; revised December 1, 2000. This work was supported in part by the National Science Council of the Republic of China under Contract NSC91-2213-E009-130 and in part by the MOE Program for Promoting Academic Excellent of Universities under Grant 89-E-FA06-2-4

T.-L. Chen is with the Department of Physics, National Central University, Taoyuan, Taiwan, R.O.C.

Y.-D. Lin is with the Institute of Communication Engineering, National Chiao Tung University, Hsinchu, Taiwan, R.O.C.

Digital Object Identifier 10.1109/TAP.2003.816312

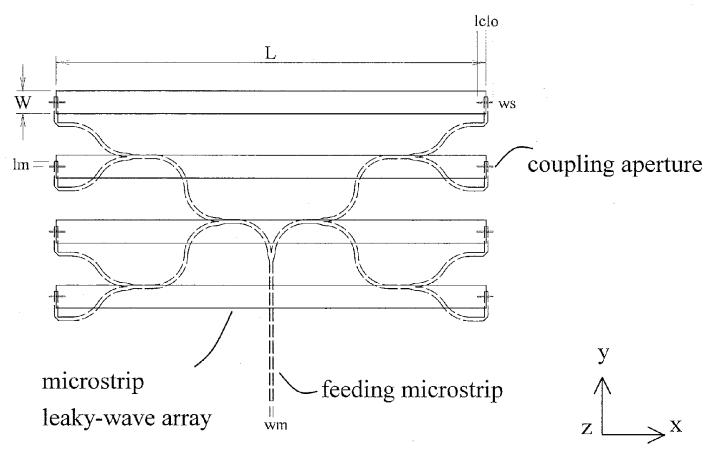

(a)

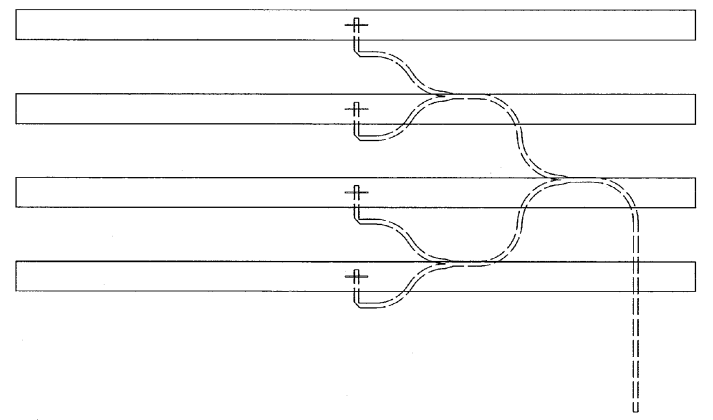

(b)

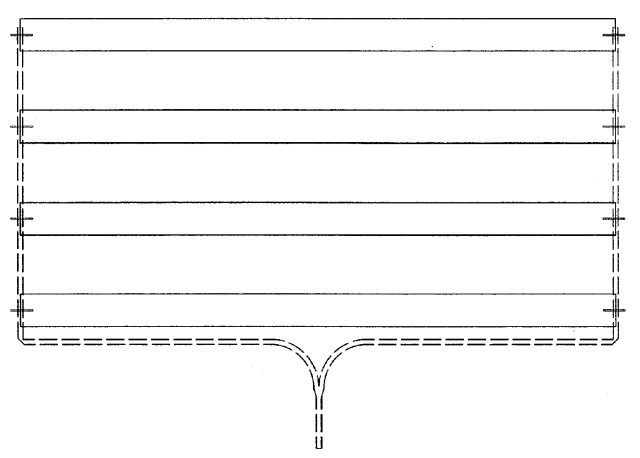

(c)

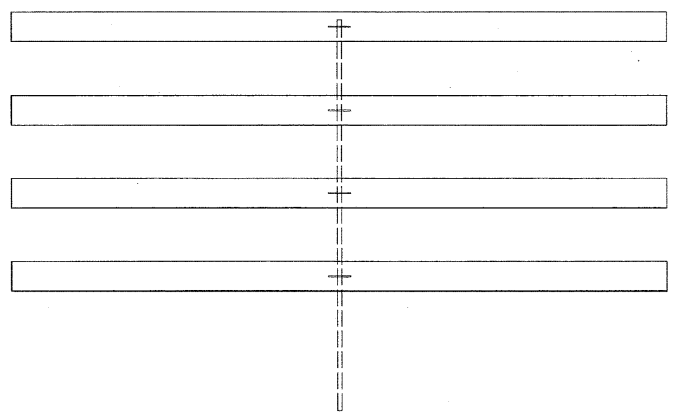

(d)

Fig. 1(a). Parallel-end-fed dual-beam aperture-coupled microstrip leaky-wave array. $\mathrm{L}=80 \mathrm{~mm}, \mathrm{w}=4.3 \mathrm{~mm}, \mathrm{lc}=1.7 \mathrm{~mm}, 1 \mathrm{o}=1.3 \mathrm{~mm}, \mathrm{ws}=0.2$ $\mathrm{mm}, \varepsilon_{\mathrm{r} 1}=2.2, \varepsilon_{\mathrm{r} 2}=10.2, \mathrm{~h}_{1}=0.508 \mathrm{~mm}, \mathrm{~h}_{2}=0.635 \mathrm{~mm}, \mathrm{wm}=0.68$ $\mathrm{mm}, 1 \mathrm{~m}=1 \mathrm{~mm}$, element space $=7.8 \mathrm{~mm}$. (b) Parallel-center-fed dual-beam aperture-coupled microstrip leaky-wave array. $\mathrm{L}=100 \mathrm{~mm}$, slot length $=$ $3.4 \mathrm{~mm}$. (c) Series-end-fed dual-beam aperture-coupled microstrip leaky-wave array. (d) Series-center-fed dual-beam aperture-coupled microstrip leaky-wave array. 


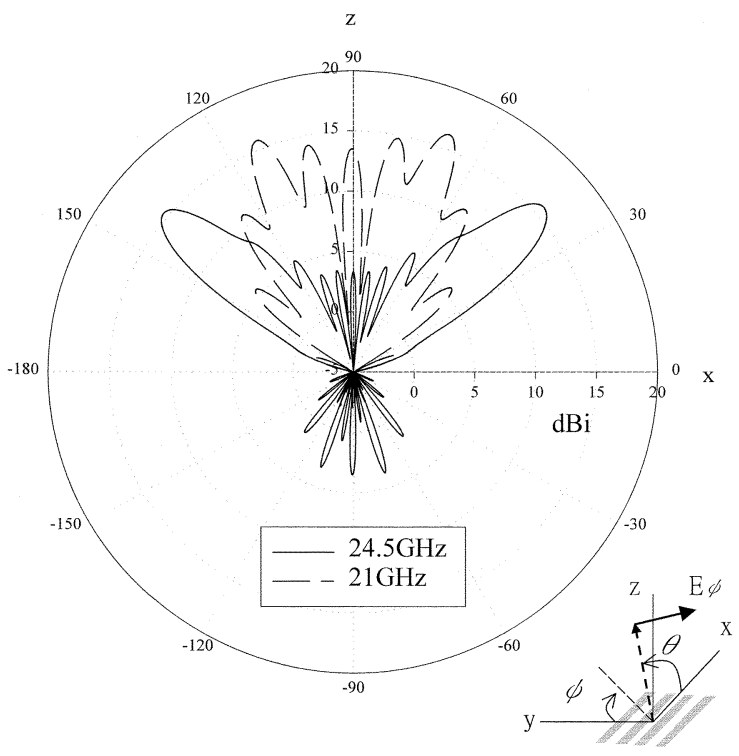

(a)

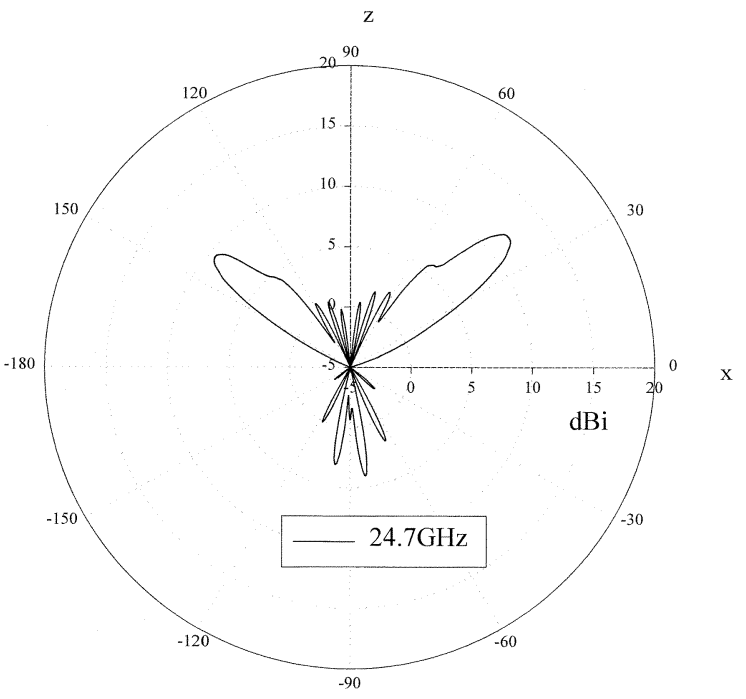

(c)

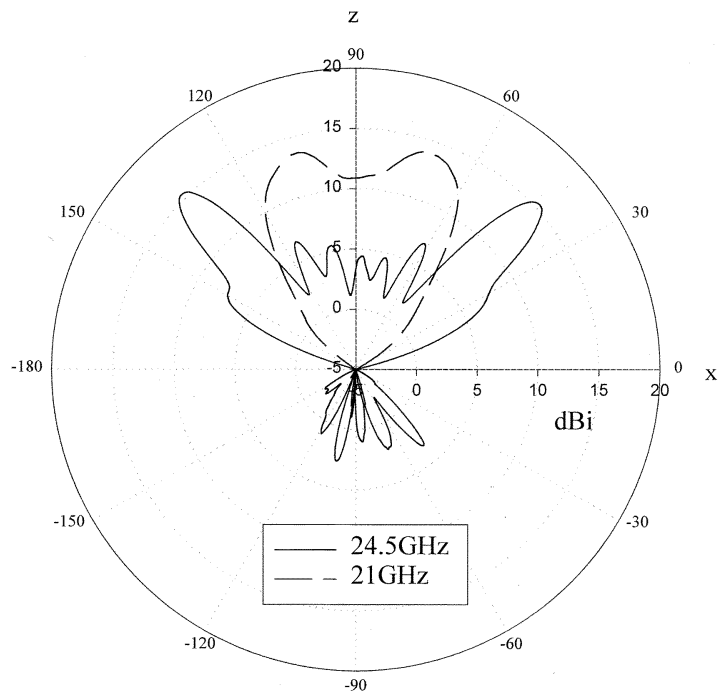

(b)

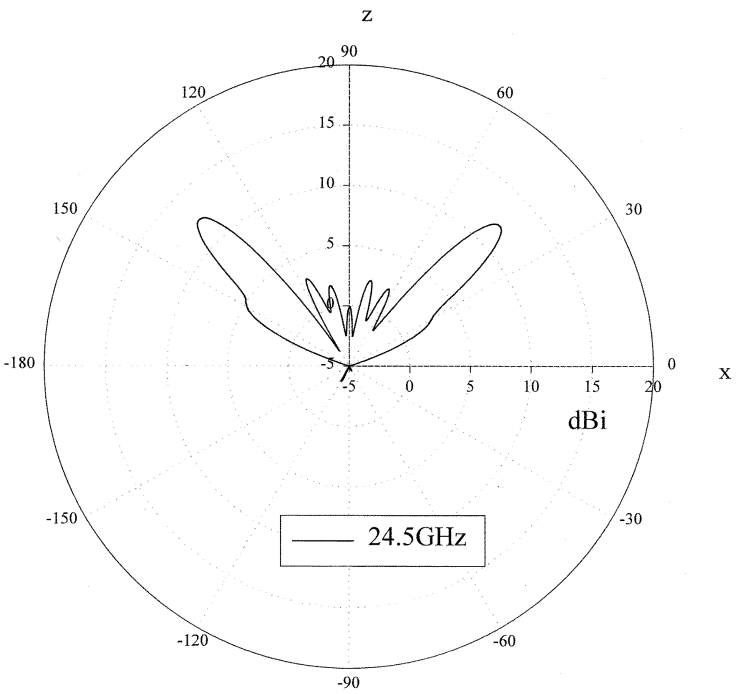

(d)

Fig. 2(a). (a) Measured $\mathrm{H}$ plane $E_{\phi}$ antenna power gain patterns of the dual-beam microstrip leaky-wave array in Fig. 1(a). (b) Measured $H$ plane $E_{\phi}$ antenna power gain patterns of the dual-beam microstrip leaky-wave array in Fig. 1(b). (c) Measured $\mathrm{H}$ plane $E_{\phi}$ antenna power gain pattern of the dual-beam microstrip leaky-wave array in Fig. 1(c). (d) Measured H plane $E_{\phi}$ antenna power gain pattern of the dual-beam microstrip leaky-wave array in Fig. 1(d).

network denoted by a dashed line is arranged on the second layer. The spacings between elements are equal and optimized to fulfill the maximal antenna gain, which is estimated by the waveguide model [8]. Two kinds of substrates are used here. Duroid laminate with lower dielectric constant $\left(\varepsilon_{r}=2.2, h=0.508 \mathrm{~mm}\right)$ is adopted for the leaky-mode microstrip antenna layer and the higher dielectric constant substrate ( $\varepsilon_{r}=10.2, h=0.635 \mathrm{~mm}$ ) for the feeding networks. This arrangement will raise the antenna efficiency and reduce the layout area of the feeding networks.

Four kinds of the feeding networks are (a) parallel-end fed, (b) parallel-center fed, (c) series-end fed, and (d) series-center fed. For (a) and (c), the dual beams are excited from both ends of the microstrips; while (b) and (d) excite the leaky mode from the center of the microstrip as the structure described in [5]. The parallel-feeding methods ((a), (b)) can easily achieve the desired power division and the phase distribution. Here, equal power dividers with modified quarter-wave transformers are used to implement the equal-amplitude and the equal-phase distribution. The series-feeding methods ((c), (d)) has simpler feeding networks than the parallel-feeding methods ((a), (b)). And no power divider is even required for center-feeding method ((d)). Since the leaky microstrips are used as the same radiator for both feeding ends of the end-feeding methods ((a), (c)), they are shorter than the leaky microstrips of the center-feeding methods ((b), (d)). Nevertheless, the end-feeding structure needs more power dividers than the center-feeding structure.

The microstrip first higher order leaky mode travels along the microstrip with an exponentially attenuating field $E_{0} e^{-j \beta x} e^{-\alpha x}$ under the strip, where $\beta$ and $\alpha$ are the phase and attenuation constants of the leaky mode [9]. The far field can be calculated by the equivalence magnetic current $\mathbf{M}=\mathbf{E} \times \mathbf{n}$, and the direction of the radiation beam 
can be roughly estimated in the direction of $\theta=\cos ^{-1}\left(\beta / k_{0}\right)$, where $\theta$ is the elevation angle from the microstrip, and $k_{0}$ is the free space wavenumber. The propagation constants can be derived by solving the spectral domain integral equations with appropriate integral contour of the inverse Fourier transform and the moment method numerical technique [10]. The shape of the coupling aperture must be properly designed in order to effectively excite the leaky mode. On the interface between the two layers the distribution of the equivalence magnetic current on the aperture should be consistent with the field of the boundary condition of the leaky mode. Narrow slot can satisfy this aperture boundary condition. The slot length is basically shorter than half wavelength of the slot mode to prevent resonance. The length of an open stub of the exciting microstrip is about one quarter of the guide wavelength and is capable of serving as another matching circuit to adjust the imaginary part of the input impedance.

\section{EXPERIMENT RESULTS}

Fig. 2 shows the measured $\mathrm{H}$ plane $E_{\phi}$ antenna power gain patterns of the arrays in Fig. 1. $E_{\phi}$ on $\mathrm{H}$ plane in this coordinate system is the dominant radiation field. The parallel-fed antennas ((a), (b)) have peak power gain of $15.4 \mathrm{dBi}$, which is roughly half of the gain of the end-fed single-beam array described in [8]. In general, to obtain high antenna gain by conventional patch array, a large number of patches with complicated power dividers and matching networks are required [11]. With the frequency varying from $21 \mathrm{GHz}$ to $24.5 \mathrm{GHz}$, the angle between the peaks of the dual beams spreads from $38^{\circ}$ to $72^{\circ}$ for the parallel-fed array. The nulls of the pattern of the end-fed array ((a), (c)) are more pronounced than that of the center-fed array $((b),(d))$. This is because of the field superposition effects from the different source pairs whose current distributions are in different exponentially decaying directions.

In the series-fed experiments (c) and (d), the same coupling apertures are used. Power excited by every aperture is different so that the current distribution on every radiating element is different. This causes the measured antenna gains of (c) and (d) to be less than those in the parallel-fed cases (a), (b). For in-phase excitation in the series-fed cases (c) and (d), the length of the feeding microstrip between apertures must be taken to be the multiples of its guide wavelength. Under this condition, the bandwidth of the series-fed type is narrower than that of the parallel-fed type, and therefore limits the beam-scanning capability. Comparing these measurements, some back radiation (to $-\mathrm{z}$ direction) resulting from the feeding circuits is found. This back radiation is smallest in the series-center fed array (d) that adopts the simplest feeding circuits.

\section{CONCLUSION}

This work describes the designs of the microstrip leaky-wave array excited by the aperture-coupling technique. A merit of the aperturecoupling method is its extra degree of freedom in designing feeding networks. By utilizing the versatile feeding arrangements on the different layers, center-, end- and parallel-, series-fed four-element dual-beam arrays are implemented. Experimental results confirm that the designs of the feeding structures can achieve practical wireless applications.

\section{REFERENCES}

[1] B. Zimmermann, W. Wiesbeck, and J. Kehrbeck, "24 GHz microwave closed-range sensors for industrial measurement applications," Microw. J., pp. 228-238, May 1996.

[2] W. Menzel, "A new traveling-wave antenna in microstrip," Archie fur Electronik und Ubertranungstechnik, vol. 33, pp. 137-140, Apr. 1979.

[3] C.-N. Hu and C.-K. C. Tzuang, "Microstrip leaky-mode antenna array," IEEE Trans. Antennas Propagat., vol. 45, pp. 1698-1699, Nov. 1997.
[4] C. Luxey and J.-M. Laheurte, "Dual-beam array of microstrip leaky-wave antennas," Electron. Lett., vol. 34, no. 11, pp. 1041-1042, May 1998.

[5] T.-L. Chen and Y.-D. Lin, "Aperture-coupled microstrip line leaky wave antenna with broadside mainbeam," Electron. Lett., vol. 34, no. 14, pp. 1366-1367, Jul. 1998.

[6] D. M. Pozar, "Microstrip antenna aperture-coupled to a microstripline," Electron. Lett., vol. 21, no. 2, pp. 49-50, Jan. 1985.

[7] D. M. Pozar and D. H. Schaubert, Microstrip Antennas, the Analysis and Design of Microstrip Antennas and Arrays. New York: IEEE Press, ch. 5.

[8] T.-L. Chen and Y.-D. Lin, "A K-band aperture-coupled microstrip leaky-wave antenna," IEICE Trans. Electron., pp. 1236-1241, July 1999.

[9] A. A. Oliner and K. S. Lee, "The nature of the leakage from higher modes on microstrip line," in IEEE MTT-S. Dig., 1986, pp. 57-60.

[10] Y.-D. Lin and J.-W. Sheen, "Mode distinction and radiation efficiency analysis of planar leaky-wave line source," IEEE Trans. Microwave Theory Tech., vol. 45, pp. 1672-1680, Oct. 1997.

[11] J. Huang and S. N. Madsen, "A dual-beam microstrip array antenna," in Proc. IEEE Antennas and Propagation Soc. Int. Symp. Dig., Chicago, IL, July 1992, pp. 147-1145.

\section{Improved Compensation for the Mutual Coupling Effect in a Dipole Array for Direction Finding}

\author{
H. T. Hui
}

\begin{abstract}
A new and practical method is proposed to compensate for the mutual coupling effect in a dipole array deployed for direction finding. This method does not require the known current distributions on the antenna elements or the known elevation angles of the incoming signals. A new definition of mutual impedance is introduced to characterize the effect due to mutual coupling between dipole elements. The new mutual impedances are calculated based on an estimated current distribution. It is shown that current method has a significantly better ability to compensate for the mutual coupling effect than previous methods. Computer simulations using the MUSIC algorithm are provided to demonstrate this method.
\end{abstract}

Index Terms-Dipole array, MUSIC algorithm, mutual coupling effect, mutual impedance.

\section{INTRODUCTION}

Direction finding is an important function of an antenna array. However, it was shown that typical eigenstructure-based direction finding algorithms, such as MUSIC, require accurate knowledge of the received signal voltages from antenna terminals as inputs [1]. This places a critical requirement on an antenna array for direction finding. This requirement is especially difficult to meet for a dipole antenna array due to the strong mutual coupling effect between the dipole elements. There have been many methods suggested to identify or to compensate for the mutual coupling effect in dipole antenna arrays. In [2], the authors used the concept of mutual impedance to derive the open-circuit voltages from the terminal voltages and showed that mutual coupling has a significant effect on the performance of adaptive arrays. This method was later used in [3] to compensate for the mutual coupling

Manuscript received November 13, 2001; revised September 21, 2002.

The author is with the Electrical and Electronic Engineering Department, Nanyang Technological University, Singapore 639798, Singapore (e-mail: ehthui@ntu.edu.sg).

Digital Object Identifier 10.1109/TAP.2003.816303 